\title{
Ablation dynamics of subsequent thermal doses delivered to previously heat-damaged tissue during magnetic resonance-guided laser-induced thermal therapy
}

\author{
Sean M. Munier, BS, Eric L. Hargreaves, PhD, Nitesh V. Patel, MD, and Shabbar F. Danish, MD \\ Division of Neurosurgery, Rutgers University, Robert Wood Johnson Medical School, New Brunswick, New Jersey
}

\begin{abstract}
OBJECTIVE Intraoperative dynamics of magnetic resonance-guided laser-induced thermal therapy (MRgLITT) have been previously characterized for ablations of naive tissue. However, most treatment sessions require the delivery of multiple doses, and little is known about the ablation dynamics when additional doses are applied to heat-damaged tissue. This study investigated the differences in ablation dynamics between naive versus damaged tissue.
\end{abstract}

METHODS The authors examined 168 ablations from 60 patients across various surgical indications. All ablations were performed using the Visualase MRI-guided laser ablation system (Medtronic), which employs a 980-nm diffusing tip diode laser. Cases with multiple topographically overlapping doses with constant power were selected for this study. Single-dose intraoperative thermal damage was used to calculate ablation rate based on the thermal damage estimate (TDE) of the maximum area of ablation achieved $\left(T D E_{\max }\right)$ and the total duration of ablation $\left(\mathrm{t}_{\max }\right)$. We compared ablation rates of naive undamaged tissue and damaged tissue exposed to subsequent thermal doses following an initial ablation. RESULTS TDE $E_{\max }$ was significantly decreased in subsequent ablations compared to the preceding ablation (initial ablation $227.8 \pm 17.7 \mathrm{~mm}^{2}$, second ablation $164.1 \pm 21.5 \mathrm{~mm}^{2}$, third ablation $124.3 \pm 11.2 \mathrm{~mm}^{2} ; \mathrm{p}=<0.001$ ). The ablation rate of subsequent thermal doses delivered to previously damaged tissue was significantly decreased compared to the ablation rate of naive tissue (initial ablation $2.703 \mathrm{~mm}^{2} / \mathrm{sec}$; second ablation $1.559 \mathrm{~mm}^{2} / \mathrm{sec}$; third ablation $1.237 \mathrm{~mm}^{2} / \mathrm{sec}$; fourth ablation $1.076 \mathrm{~mm} / \mathrm{sec} ; p=<0.001$ ). A negative correlation was found between $T D E_{\max }$ and percentage of overlap in a subsequent ablation with previously damaged tissue $(r=-0.164 ; p<0.02)$.

CONCLUSIONS Ablation of previously ablated tissue results in a reduced ablation rate and reduced $\mathrm{TDE}_{\max }$. Additionally, each successive thermal dose in a series of sequential ablations results in a decreased ablation rate relative to that of the preceding ablation. In the absence of a change in power, operators should anticipate a possible reduction in TDE when ablating partially damaged tissue for a similar amount of time compared to the preceding ablation.

https://thejns.org/doi/abs/10.3171/2018.7.JNS18886

KEYWORDS magnetic resonance-guided laser thermal therapy; magnetic resonance thermometry; thermal ablation; MRgLITT

$\mathrm{M}$ AGNETIC resonance-guided laser-induced thermal therapy (MRgLITT) is a minimally invasive surgical procedure used in the treatment of various intracranial pathologies. The technique utilizes thermal energy delivered through a fiber optic laser catheter to achieve focal ablation of intracranial neoplasms. ${ }^{22}$ The procedure is performed under the guidance of magnetic resonance thermal imaging (MRTI), allowing for tight control of the target area while minimizing undesired damage to surrounding healthy tissue. ${ }^{18,22}$ The ability to closely monitor therapy in real time coupled with a stereotactic approach has brought about a renewed interest in the technique as a potential tool in the treatment of difficult-to-treat intracranial pathologies. ${ }^{2,4,14}$ Previous studies have examined MRgLITT as a potential treatment option for a diverse range of intracranial pathologies, including glioblastoma multiforme (GBM) ${ }^{12}$ ependymomas, ${ }^{12}$ epilepsy, ${ }^{7,8,10,35}$ metastatic tumors, ${ }^{12,25,32}$ and intractable pain. ${ }^{28}$ Nonetheless, the ability to answer some fundamental questions will improve the procedure. In this study, we attempt

ABBREVIATIONS GBM = glioblastoma multiforme; GLM = generalized linear model; MRgLITT = magnetic resonance-guided laser-induced thermal therapy; MRTI = magnetic resonance thermal imaging; TDE $=$ thermal damage estimate; $t_{\max }=$ ablation duration.

SUBMITTED April 1, 2018. ACCEPTED July 31, 2018.

INCLUDE WHEN CITING Published online December 21, 2018; DOI: 10.3171/2018.7.JNS18886. 

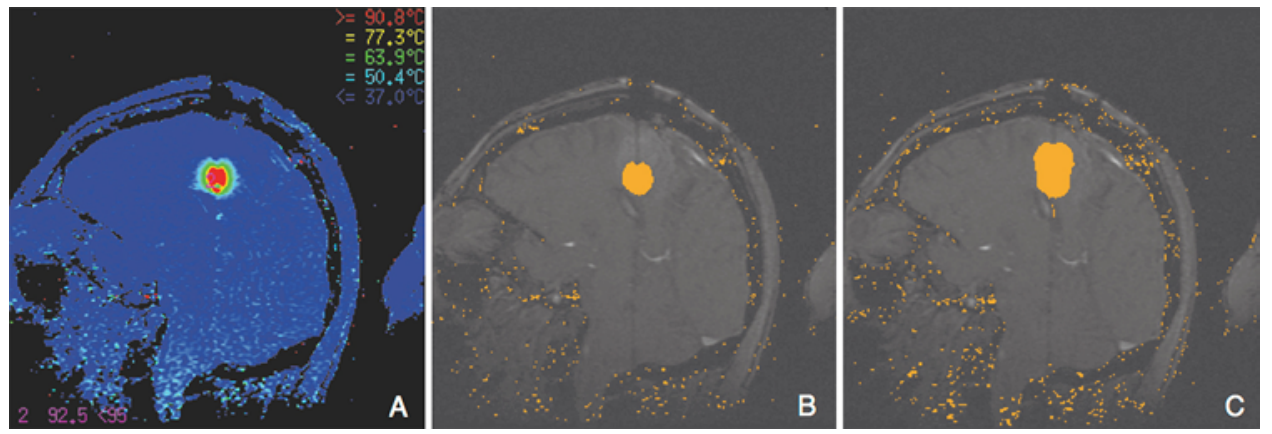

FIG. 1. A and B: Example of an ablation with real-time magnetic resonance thermal imaging $(A)$ and thermal damage estimate (B). C: Image depicting $T D E_{\max }$ of 3 subsequent ablations superimposed over one another in a case requiring 3 thermal doses. Figure is available in color online only.

to answer the question, "what happens when the ablation is performed in tissue overlapping with that which was just ablated?"

To date, several studies have evaluated ablation dynamics in the context of different intraoperative variables, thereby expanding the current understanding of intracranial ablation patterns. Important operative parameters subject to variation by the surgeon include laser power, temperature boundaries, and ablation duration..$^{30}$ Previously, the temporal profile of neural tissue damage via laser energy has been characterized through assessment of the relationship between thermal damage estimate (TDE) and ablation duration $\left(\mathrm{t}_{\max }\right)$, finding that expansion of the ablation area can follow either logistic or negative exponential growth as it approaches a finite maximum $\left(\mathrm{TDE}_{\mathrm{max}}\right)$. $^{21,30}$ A second study investigated the effects of variable laser power on ablation duration and ablation area, finding that the degree of power can both hasten and enlarge a given ablation. ${ }^{21}$ However, it is critical to note that most treatment sessions require the delivery of multiple thermal doses from the laser catheter to achieve full target ablation, and little is known about the ablation dynamics when additional doses are applied to previously heat-damaged tissue. In cases with large or irregularly shaped targets, the operator can retract the laser catheter along the longitudinal axis of the lesion between ablations and apply a second thermal dose to expand the ablation area. Depending on lesion size and shape, complete target destruction may require the use of as many as 4 to 5 overlapping thermal doses within the same operative session. As such, further research is required to understand whether the principles guiding ablation of naive tissue vary from those that apply to subsequent ablations. This study examines how the application of laser energy to previously damaged tissue affects ablation rate, ablation duration, and ablation area in the absence of a change in laser power. To our knowledge, this is the first report in the literature to examine ablation dynamics of previously heat-damaged tissue undergoing multiple topographically overlapping ablations at constant power within a single operative session.

\section{Methods}

\section{Patient Selection}

We retrospectively reviewed and selected a total of 60 patients who underwent MRgLITT for various intracranial pathologies at our institution and evaluated 168 total thermal doses across the patient population. Each selected patient underwent 2 or more overlapping thermal doses within a single operative session. Pathology and patient demographics were not part of the selection criteria for this study. All patients underwent the procedure as part of the routine clinical care algorithm at our institution and were enrolled in an institutional review board-approved protocol.

\section{Laser Ablation Procedure}

The laser ablation procedure has previously been described. Briefly, each patient is subjected to preoperative $\mathrm{CT}$ and MRI. The images are loaded onto stereotactic planning software and fused to facilitate planning of catheter trajectory. ${ }^{12}$ Stereotactic placement of the laser is accomplished using bone-implanted fiducials for registration. Using intraoperative neuro-navigation, a small burr hole is created using a 3.2-mm twist drill. Following fixation of a Visualase Thermal Therapy System bone anchor to the calvaria, the laser catheter is introduced through the anchor to its predetermined trajectory length. The patient is transferred to the MRI suite where the ablation procedure is performed.

The procedure is performed under the guidance of MRTI, allowing for real-time thermal monitoring of the ablation procedure (Fig. 1A). Using the Visualase software, the operator first identifies safety margins to prevent steam and pressure accumulation, such that the peripheral tissue temperature does not surpass $50^{\circ} \mathrm{C}$ and that the target center temperature does not surpass $90^{\circ} \mathrm{C}$. The procedure begins with the delivery of a short low-power test dose to confirm accurate laser placement. The operator can then initiate treatment at a user-determined power with the goal of achieving maximal target ablation. The laser is cooled throughout the procedure via an enclosed saline irrigating catheter system, which promotes uniform dispersal of thermal energy. ${ }^{12}$ The ablation area extends from the laser catheter in an ellipsoid fashion, with the rate of growth in any one direction varying based on several factors, such as target morphology and tissue composition. ${ }^{16}$ The operator monitors the ablation area in real time through interpretation of the system-generated TDE, 
which provides a computational estimate of target ablation area every 3-5 seconds (Fig. 1B). The system software generates the TDE using the Arrhenius rate model, which uses tissue temperature and duration maintained at a specific temperature as primary variables. ${ }^{24,30}$ In cases for which the target cannot be fully destroyed by a single thermal dose due to size or irregular shape, the surgeon can retract the laser catheter along the longitudinal axis of the target between laser doses to expand the ablation area. Furthermore, adjustments to laser power and irrigation rate can be made by the surgeon between doses if deemed necessary. Any additional ablative area generated by subsequent laser ablations delivered after the initial ablation can be and is usually superimposed over the TDE produced by the previous thermal dose (Fig. 1C).

\section{Data Analysis}

In each case considered, power was kept constant in all thermal doses delivered. Power across the 60 cases ranged from 8 to $15 \mathrm{~W}$. To estimate the area of ablation in each successive TDE image, we counted the number of superimposed color-coded pixels in each frame (JPG format) using an image processing software, GNU Image Manipulation Program version 2.8.4 (Free Software Foundation). The field of view was set at $240 \times 240 \mathrm{~mm}^{2}$ in each of the 60 cases analyzed. Each pixel corresponds to $0.9 \mathrm{~mm}^{2}$. To calculate ablation rate for thermal doses delivered after the initial ablation, the TDE was reset to zero between ablations to isolate the TDE produced by each independent thermal dose. Percent overlap was calculated by dividing the area of the previous ablation contained within the area generated by the subsequent ablation by the total area of the subsequent ablation. Figure 2 further depicts the data analysis process.

\section{Statistical Analysis}

All statistical analyses were performed using IBM SPSS version 24 (IBM Corp.). A p value $<0.05$ was used to determine statistical significance. The data were analyzed using a series of generalized linear model (GLM) repeated measures analyses. An additional analysis was performed using a between-groups analysis to verify the relationship found in the GLM repeated measures analysis. Further analyses were performed to analyze the effects of power and degree of overlap on ablation duration and $\mathrm{TDE}_{\max }$. A final GLM ANCOVA was performed with the covariates of laser power and percentage overlap to analyze the effects of these intraoperative variations on ablation rate.

\section{Results}

In 60 patients, 60 LITT cases requiring at least 2 thermal doses to extend the induced lesion to the desired boundaries during a single session were examined. Of the 60 cases, 34 required 2 ablations, 15 required 3 ablations, and 11 cases required 4 or more ablations, with 2 of those cases requiring 5 and 6 ablations each, respectively. In this population, no serious adverse events were recorded. Patient pathology and demographics are summarized in Table 1.
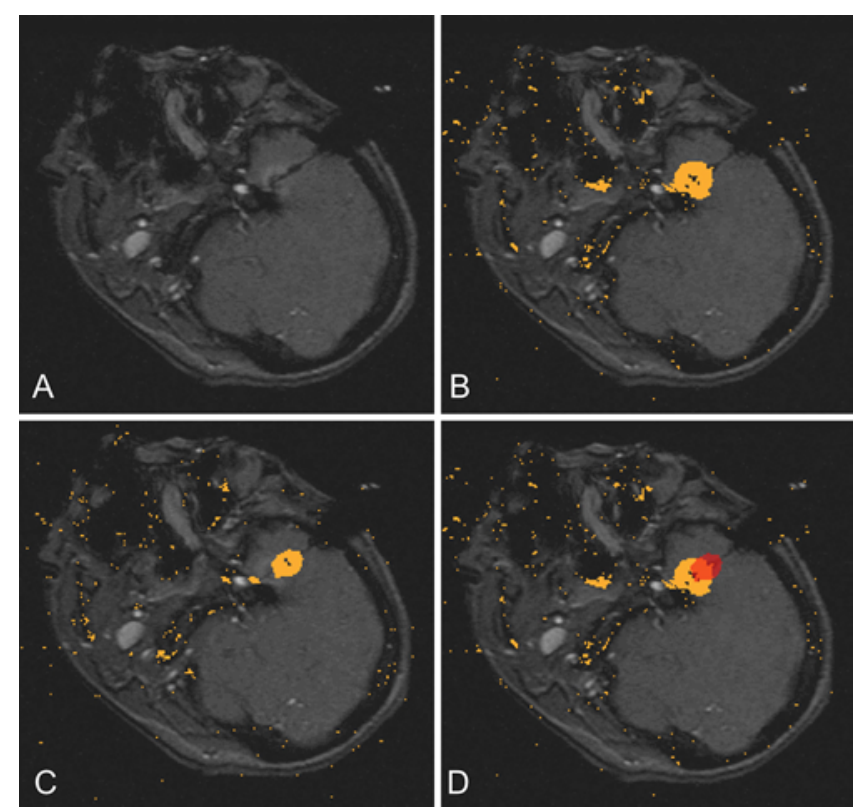

FIG. 2. Example of a patient with a left temporal metastatic tumor with the laser catheter in place before administration of 2 thermal doses $(\mathbf{A})$. Ablation area produced by the first thermal dose (B) and second thermal dose (C). The orange pixelated area represents ablative area and is reset between each thermal dose such that the individual ablative area produced by each thermal dose can be analyzed separately. Ablation rate is calculated by counting the number of orange pixels overlaying the image, which is then converted to area in square millimeters and divided by the ablation duration. D: Ablation areas of the 2 thermal doses superimposed over one another with the ablative area produced by the second thermal dose shown in translucent red. Area of overlap is calculated by tracing the overlapping area of the 2 TDEs and dividing by the area found in $\mathrm{C}$. Figure is available in color online only.

A series of GLM repeated measures analyzed the ablation rate of subsequent thermal doses delivered during a single session (Fig. 3A). Of those 34 cases requiring only 2 ablations, the mean ablation rate for the initial lesion was $2.424 \mathrm{~mm}^{2} / \mathrm{sec}$ (SEM 0.235), while the average ablation rate for the second lesion was $1.724 \mathrm{~mm}^{2} / \mathrm{sec}$ (SEM 0.220). Results indicated a decline in ablation rate between the first and second ablations $\left(\mathrm{F}_{(1.33)}=54.579 ; \mathrm{p}<0.001\right)$. For those 15 cases requiring 3 ablations, the average ablation rates for the first, second, and third lesions were 2.035 $\mathrm{mm}^{2} / \mathrm{sec}(\mathrm{SEM}=0.252), 1.488 \mathrm{~mm} / \mathrm{sec}(\mathrm{SEM}=0.237)$, and $1.309 \mathrm{~mm}^{2} / \mathrm{sec}(\mathrm{SEM}=0.204)$, respectively. Results of this analysis indicated a strong linear decline in ablation rate across all 3 lesions $\left(\mathrm{F}_{(1,14)}=11.396 ; \mathrm{p}=0.005\right)$. A final repeated measures analysis on the subset of 11 cases requiring 4 ablations also found a strong downward linear trend for ablation rate across the lesions $\left(\mathrm{F}_{(1,10)}=17.484 ; \mathrm{p}\right.$ $=0.002$ ), with the average ablation rate for the first, second, third, and fourth lesions being $2.738 \mathrm{~mm}^{2} / \mathrm{sec}$ (SEM $=0.334), 2.059 \mathrm{~mm}^{2} / \mathrm{sec}(\mathrm{SEM}=0.242), 1.641 \mathrm{~mm}^{2} / \mathrm{sec}$ $(\mathrm{SEM}=0.189)$, and $1.405 \mathrm{~mm}^{2} / \mathrm{sec}(\mathrm{SEM}=0.164)$, respectively.

GLM repeated measures analyses parallel to those above were performed on both the ablation duration and resulting ablative area. In cases requiring 2 or 3 thermal 
TABLE 1. Patient information and operative indications

\begin{tabular}{lc}
\hline \multicolumn{1}{c}{ Characteristic } & $\begin{array}{c}\text { No. }(\%) \text { of Patients } \\
(\mathrm{n}=60)\end{array}$ \\
\hline Age group, yrs & $0(0.0)$ \\
\hline$<18$ & $13(21.7)$ \\
\hline $18-44$ & $25(41.7)$ \\
\hline $45-64$ & $22(36.6)$ \\
\hline$\geq 65$ & \\
\hline Sex & $29(48.3)$ \\
\hline Male & $31(51.7)$ \\
\hline Female & $1(1.7)$ \\
\hline Indication for ablation & $11(18.3)$ \\
\hline CNS lymphoma & $8(13.3)$ \\
\hline Epilepsy & $5(8.3)$ \\
\hline GBM & $1(1.7)$ \\
\hline Intractable pain & $8(13.3)$ \\
\hline Primary glioma & $4(6.7)$ \\
\hline Recurrent GBM & $21(35)$ \\
\hline Recurrent glioma & $1(1.7)$ \\
\hline Recurrent metastasis/radiation necrosis & \\
\hline Teratoma & \\
\hline
\end{tabular}

doses, there was no statistical difference in ablation duration across thermal doses (Fig. 3B). However, a statistically significant linear increase was seen in cases requiring 4 thermal doses (Table 2). For $\mathrm{TDE}_{\max }$, a significant linear decrease in ablative area was seen in cases requiring 2, 3, or 4 ablations (Table 3, Fig. 3C).

A series of control analyses were subsequently performed to determine whether the first, second, and third ablation rates were similar across the total patient population regardless of the total number of ablations a patient received. The analyses involved comparing the first ablation and second ablation rates between cases requiring 2 , 3 , and 4 ablations. This analysis was repeated once again for the third ablation rate in cases requiring 3 and 4 ablations. These control analyses showed that ablation rates for cases requiring a second, third, or subsequent fourth ablation were not different from each other as groups compared to any of the first $\left(\mathrm{F}_{(2,57)}=1.062 ; \mathrm{p}=0.353\right)$, second $\left(\mathrm{F}_{(2.57)}=0.815 ; \mathrm{p}=0.448\right)$, or third ablation rates $\left(\mathrm{F}_{(1,24)}\right.$ $=1.320 ; p=0.262$ ). Because the control analyses found no significant difference in the first, second, and third ablation rates across the total population, the ablation rate was again analyzed as a between-subjects GLM, using the entire set of 60 cases undergoing the first and second ablations as the first and second levels, while the subset of 26 cases undergoing 3 or more ablations, and final subset of 11 cases undergoing 4 ablations, were considered the third and fourth levels of an independent factor. Results from the between-subjects GLM once again showed linear statistical differences on ablation rate across the 4 lesions represented as levels $\left(\mathrm{F}_{(3,153)}=6.688 ; \mathrm{p}<0.001\right)$ (Fig. 4).

Previous studies have shown that laser power (watts) contributes to the overall ablation rate. ${ }^{21}$ Although laser
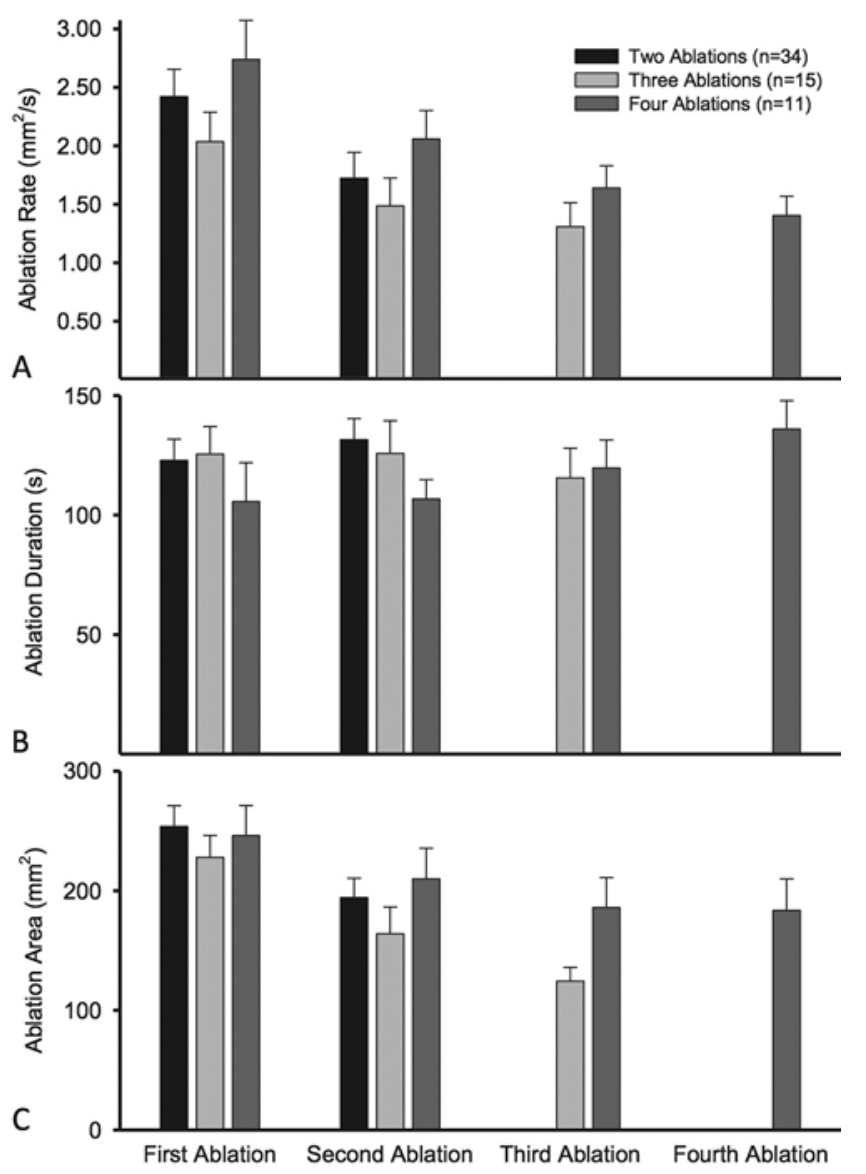

FIG. 3. Bar graphs of data for cases requiring 2, 3, or 4 ablations, with SEM (vertical lines) following GLM repeated measures analysis. The shaded bars represent the different groups that underwent repeated measures analysis based on the number of total thermal doses delivered in each specific case (either 2, 3, or 4 thermal doses). A: Ablation rates. $B$ : The duration required to reach $\mathrm{TDE}_{\max }$ in cases requiring 2, 3, or 4 ablations, with SEM (vertical lines) following GLM repeated measures analysis. C: Ablation area of cases requiring 2, 3, and 4 ablations with SEM (vertical lines) following GLM repeated measures analysis.

power was held constant for successive ablations within each case, it varied between cases. It was further hypothesized that a higher percentage of area overlap between ablations may have slowed subsequent ablations. Correlational analyses verified the relationships between laser power $\left(\mathrm{r}_{(155)}=0.323 ; \mathrm{p}<0.001\right)$, percentage area of overlap $\left(r_{(155)}=-0.164 ; p=0.02\right)$, and ablation rate.

Consequently, these factors were added to the lattermost GLM ANCOVA to see if they could potentially ameliorate or explain the decline over successive ablation rates. Both laser power $\left(\eta_{\mathrm{p}}^{2}=0.115 ; \mathrm{p}<0.001\right)$ and percentage area of overlap $\left(\eta_{\mathrm{p}}{ }^{2}=0.030 ; \mathrm{p}=0.034\right)$ returned statistically significant eta-squared values, indicating contributions to the model. The final analysis, which included the 2 covariates of laser power and percentage area of overlap, revealed an enhanced linear effect of declining ablation rate on subsequent ablations $\left(\mathrm{F}_{(3,151)}=7.303 ; \mathrm{p}<0.001\right)$, with estimated adjusted means of $2.703 \mathrm{~mm}^{2} / \mathrm{sec}$ (SEM = $0.191), 1.559 \mathrm{~mm}^{2} / \mathrm{sec}(\mathrm{SEM}=0.149), 1.237 \mathrm{~mm}^{2} / \mathrm{sec}(\mathrm{SEM}$ 
TABLE 2. $t_{\max }$ for cases requiring 2,3 , and 4 thermal doses

\begin{tabular}{|c|c|c|c|c|c|c|}
\hline No. of Thermal Doses & Ablation $1 \mathrm{t}_{\max }(\mathrm{sec})$ & Ablation $2 \mathrm{t}_{\max }(\mathrm{sec})$ & Ablation $3 t_{\max }(\mathrm{sec})$ & Ablation $4 \mathrm{t}_{\max }(\mathrm{sec})$ & F Value & $\mathrm{p}$ Value \\
\hline $2(n=34)$ & $122.9 \pm 8.7$ & $131.6 \pm 8.7$ & - & - & 1.888 & 0.179 \\
\hline $3(n=15)$ & $125.5 \pm 11.1$ & $125.8 \pm 13.2$ & $115.5 \pm 12.0$ & - & 0.873 & 0.366 \\
\hline $4(n=11)$ & $105.6 \pm 15.5$ & $106.7 \pm 7.7$ & $119.7 \pm 11.2$ & $136.0 \pm 11.3$ & 4.959 & 0.050 \\
\hline
\end{tabular}

Values are mean \pm SEM unless otherwise indicated.

$=0.224)$, and $1.076 \mathrm{~mm}^{2} / \mathrm{sec}(\mathrm{SEM}=0.314)$ for the first, second, third, and fourth successive ablation rates (Fig. 5).

\section{Discussion}

Over the last decade, the use of MRgLITT in the field of neurosurgery has expanded rapidly due in large part to significant advances in imaging and laser ablation software. With this growth, the efficacy of the procedure in the treatment of various intracranial pathologies has been reexamined, with more institutions adopting the technique each year. ${ }^{6,12,13,22,32}$ Nonetheless, while the effects of laser ablation on biological tissue have been studied in organ systems outside of the CNS, little has been reported on how nervous tissue reacts to the application of thermal heat for the treatment of intracranial neoplasms. Specifically, little is known on how multiple subsequent ablations of brain tissue within a single operative session affect the duration of ablation required to achieve target destruction, or how previous ablations affect the maximum achievable ablation area in subsequent ablations. We performed MRgLITT using the Visualase system, which is one of the 2 FDA-approved systems for intracranial ablation. The Visualase system utilizes a $980-\mathrm{nm}$ diffusing tip diode laser, which is enclosed within a cooling catheter that acts to cool both the applicator fiber and the adjacent tissue while promoting a clean dispersion of thermal energy. ${ }^{5}$ When ablating biological tissue, the nature and extent of photothermal effects of laser-tissue interactions are governed by the distribution of light within the tissues, tissue temperature, the duration for which tissue is exposed to elevated temperature, and the tissue thermal properties of heat capacity and diffusivity. ${ }^{11,15,20,26,34}$ With these variables in mind, in the absence of a change in intraoperative parameters, any changes seen in ablation dynamics in subsequent ablations compared to ablation of naive tissue can be assumed to be attributable to changes in light distribution within the tissue and changes in tissue thermal properties following exposure to an initial thermal dose. In this study, we investigated how these changes affect the rate of tissue ablation and maximal achievable ablative area. Specifically, we compared the rates of ablation and maximal thermal damage estimates in ablations of unburned naive tissue to subsequent ablations within those cases in the absence of a change in other operative parameters such as laser power or irrigation rate. Lastly, we considered the degree of overlap between an initial ablation and subsequent ablations and examined its effect on ablation rate and area.

The three major factors to consider preoperatively when planning the laser trajectory and insertion site are lesion location, size, and shape. During the planning stage, it is critical that the laser evenly divides the target along its maximal longitudinal trajectory to allow a delivery of laser energy that facilitates complete target destruction. ${ }^{3}$ In cases with large targets or targets with irregular shapes, it is even more critical that the planning stage take these factors into consideration, as these lesions typically require multiple topographically overlapping thermal doses to ensure complete target destruction. Intraoperatively, there are two options at the surgeon's disposal for expansion of the ablative area, increasing laser power and retraction of the laser catheter along the applicator insertion trajectory. ${ }^{21}$ The laser catheter utilized by the Visualase system disperses heat in all directions in an ellipsoidal fashion. ${ }^{19}$ Previous studies have shown that in cases in which a second thermal dose at higher power is applied to a target without repositioning the laser following an initial lower power dose, the TDE expands to a larger area with an increased ablation rate. ${ }^{21}$ Our study specifically examined how ablation dynamics are affected when laser power remains constant, but laser position changes. The most notable finding within the study is that in subsequent ablation of previously heat-damaged tissue, the rate of ablation is significantly reduced compared to ablation of naive tissue, and the ablation rate continues to decline with each successive ablation. Additionally, the TDE of subsequent ablations is significantly decreased compared to that for ablation of naive tissue, while the mean duration required to reach $\mathrm{TDE}_{\max }$ remains relatively constant. It is also important to note that while the ablation rate continues to decline through the fourth thermal dose, $\mathrm{TDE}_{\max }$ was

TABLE 3. TDE ${ }_{\max }$ for cases requiring 2, 3, and 4 thermal doses

\begin{tabular}{|c|c|c|c|c|c|c|}
\hline No. of Thermal Doses & Ablation $1 \mathrm{TDE}_{\max }\left(\mathrm{mm}^{2}\right)$ & Ablation $2 \mathrm{TDE}_{\max }\left(\mathrm{mm}^{2}\right)$ & Ablation $3 \mathrm{TDE}_{\max }\left(\mathrm{mm}^{2}\right)$ & Ablation $4 \mathrm{TDE}_{\max }\left(\mathrm{mm}^{2}\right)$ & F Value & $p$ Value \\
\hline $2(n=34)$ & $253.7 \pm 17.0$ & $194.3 \pm 15.9$ & - & - & 14.438 & 0.001 \\
\hline $3(n=15)$ & $227.8 \pm 17.7$ & $164.1 \pm 21.5$ & $124.3 \pm 11.2$ & - & 59.081 & $<0.001$ \\
\hline $4(n=11)$ & $246.0 \pm 24.0$ & $209.9 \pm 24.4$ & $185.9 \pm 23.9$ & $183.5 \pm 25.2$ & 5.515 & 0.041 \\
\hline
\end{tabular}

Values are mean \pm SEM unless otherwise indicated. 


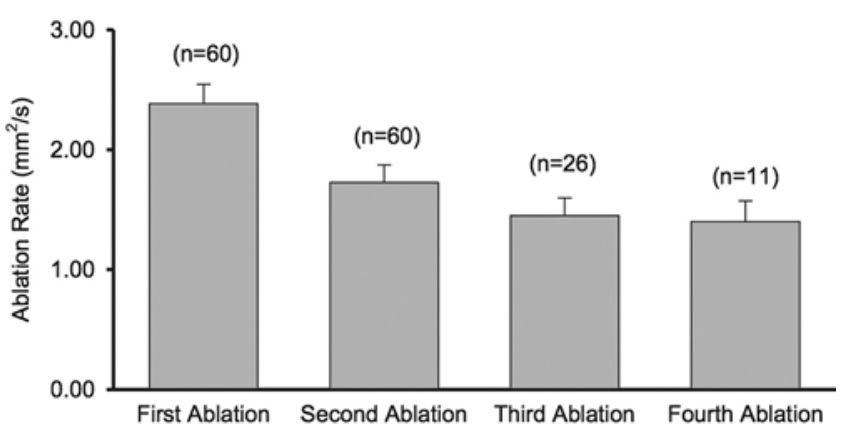

FIG. 4. Mean and SEM (vertical lines) of ablation rate as calculated as a between-groups analysis. These measures were displayed for each of the first, second, third, and fourth ablations, with outcomes collapsed across all cases.

found to level off between the third and fourth ablation, suggesting that the effects of multiple ablations become asymptotic after the third ablation with regard to area of ablation. Still, there was a statistically significant increase in ablation duration across cases requiring 4 ablations, which again reflects a decline in rate of ablation and a greater amount of time needed to ablate an area equal to the previous ablation.

Intuitively, subsequent ablations following an initial thermal dose would be expected to expand at a faster rate, as repeated doses of thermal energy are additive if delivered before tissue completely cools. ${ }^{31}$ However, the results observed in this study suggest the opposite effect. The underlying mechanism for this observation is likely multifactorial, as previous experiments have shown that the microscopic response of tissue to thermal stress is highly complex. ${ }^{33}$ Tissue exposure to temperatures between $60^{\circ} \mathrm{C}$ and $100^{\circ} \mathrm{C}$ has been shown to cause thermal denaturation and contraction of intracellular cellular proteins along with collapse of the cytoskeleton, both of which are capable of altering the thermal conductivity and specific heat of tissue. ${ }^{31}$ At temperatures greater than $100^{\circ} \mathrm{C}$, vaporization and carbonization of tissue occur, which drastically reduces the efficiency of heat distribution throughout the tissue. ${ }^{1,31}$ However, given that the Visualase system automatically deactivates when the temperature at the center of the target exceeds $90^{\circ} \mathrm{C}$, this is unlikely to play a significant role in this setting. More likely, the increased thermal capacity of a localized area of brain tissue following an initial thermal dose is related to local effects caused by increased perfusion and disruption of the blood-brain barrier. ${ }^{27}$ Specifically, thermal capacity of a given tissue is dominated by its perfusion, and regional increases in perfusion accompany regional changes in temperature. ${ }^{27}$ This proposed mechanism is supported by a previous study which found that GBM requires a longer duration of ablation to achieve complete target destruction compared with other pathologies, likely due to heat sinks within the target tissue. ${ }^{30}$ This idea of GBM vascularity acting as a heat sink is supported by a previous study that utilized MRI to evaluate relative blood volume across different tumor types, finding that GBM had significantly higher relative blood perfusion compared with anaplastic gliomas and low-grade gliomas. ${ }^{29}$ Together, these ideas suggest that

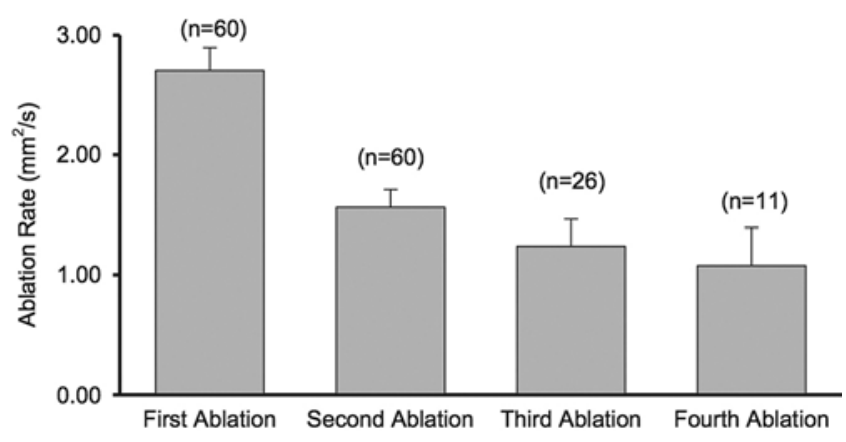

FIG. 5. Adjusted mean ablation rates calculated with SEM (vertical lines) from ANCOVA. Covariates included in the analysis were laser power and degree of ablation overlap.

increased tissue temperature caused by an initial ablation results in increased local perfusion and an overall reduction in laser ablation efficiency, thereby reducing ablation rate and ablation area. These thoughts, while speculative, may provide some insight into the possible mechanism of a reduced ablation rate following an initial ablation. Regardless of the underlying mechanism, operators should anticipate a likely reduction in ablation rate and area when subsequently ablating tissue following an initial dose for a similar amount of time at constant power. Operators should be aware that delivering a second thermal dose without changing the laser power or laser position is unlikely to result in ablation of new tissue outside of the boundaries of what was ablated during the initial ablation.

Another significant finding in this study is that the greater the degree of overlap between subsequent ablations, the greater the reduction in ablation rate during the latter thermal dose compared to the initial dose. In other words, in cases in which the laser catheter is retracted only several millimeters from the site of the initial ablation, the ablation rate of the next ablation can be expected to negatively deviate from the previous ablation to a higher degree than would occur with larger retractions of the laser catheter. In the context of the potential changes in photodynamic properties previously discussed, this finding is intuitive, as larger retractions of the laser catheter result in greater amount of naive tissue ablation. Thus, smaller decrements in ablation rate can be expected when the laser catheter is retracted a greater distance from the original ablation site, as a greater proportion of the tissue being ablated will have naive tissue thermal properties. Ablation overlap is evidently an extremely important factor in the context of ablation dynamics, as our statistical analysis found it to be the greatest contributor to variance across the different metrics examined in this study.

A number of supplemental analyses performed are also worthy of mention. The main outcome of ablation rate was evaluated as a repeated measures analysis and as an ANCOVA, both of which yielded the same significant findings of diminishing rate and ablative area with successive ablations. The consistency in results between these two different statistical assessments is significant, as it suggests that regardless of whether a patient undergoes 2,3 , or 4 thermal doses, the same trend will be observed in that 
both ablation rates will decrease after the initial ablation. Additionally, this relationship still stands when degree of overlap and laser power are factored in as covariates.

From a practical perspective, this study should guide the user to increase the power and duration of the subsequent ablation if a larger ablation volume is required, especially if there is overlap with prior ablated tissue.

There are several limitations in this study. Most notably, the ablation rates calculated in each case were treated as constant, as they were based on only 2 data points: $\mathrm{TDE}_{\text {max }}$ and total duration of ablation. Previous studies have shown that when plotted against time, the ablation area follows a negative exponential growth as it approaches a finite maximum, suggesting a diminishing rate of ablation over time..$^{21,30}$ Because our data assume a constant rate of ablation throughout the full thermal dose, it is possible that the changes in rate observed are not proportional along the full range of the ablation curve. However, because our study focuses on informing the operator approach over the course of a full thermal dose, these small intraablative variations are likely without clinical significance.

Another limitation of note relates to the setting of the cooling catheter during the operation. The system allows for the irrigation rate to be set between 1 and 10: in our study, the irrigation rate was set between levels 1 and 2 in each of the 60 cases analyzed. The cooling catheter acts as a heat sink during the ablation procedure by removing heated saline around the laser applicator tip and replacing it with cool fluid. This acts to increase the depth of thermal energy penetration by preventing local effects to the area immediately adjacent to the laser applicator that would reduce energy dispersion efficiency. ${ }^{9}$ In the context of discussing photothermal tissue properties, irrigation rate may potentially be an important factor to consider, though at this time it is difficult to quantitate the effect of irrigation speed on temperature and ablation dynamics. Moving forward, there will need to be some attention paid to this variable, as it can be adjusted by the user during the procedure. ${ }^{28}$

A final limitation of this study is that the data reported were acquired using the Visualase MRI-guided laser ablation technology. The Monteris NeuroBlate ablation system is another system approved for intracranial ablation; however, this system utilizes a different catheter design, thermal damage threshold temperature, and laser wavelength. As such, the parameters and conclusions reported here may not be applicable to the NeuroBlate system. It is important to mention that the findings reported within this article are dependent on the accuracy of the Visualase software in producing reliable and consistent estimates of thermal ablative area. To date, several notable studies have sought to verify this accuracy. McNichols et al. first investigated the accuracy of the TDE in canine cerebral tissue by evaluating histopathological specimens, finding a strong correlation between the LITT-predicted ablation extent and postoperative histopathological tissue analysis. ${ }^{17}$ This conclusion was further supported in a study that evaluated whether the TDE correlates with ablative area seen on postoperative MRI in perfused intracranial pathologies, once again finding that the TDE is in fact an accurate and reliable measure of ablative area. ${ }^{23}$ Based on these studies, we can be confident that the findings reported within this study are based on consistent and reliable data.

\section{Conclusions}

Ablation of previously heat-damaged tissue results in a reduction of ablation rate and ablation area. Additionally, each successive thermal dose in a series of sequential ablations results in a decreased ablation rate relative to that of the preceding ablation. Larger reductions in ablation rate are associated with higher degrees of overlap between sequential thermal doses. In the absence of a change in power, operators should anticipate a possible reduction in TDE when ablating partially damaged tissue for a similar amount of time compared to the preceding ablation. Future studies are needed to investigate the effect of irrigation rate on ablation dynamics.

\section{References}

1. Ansari MA, Erfanzadeh M, Mohajerani E: Mechanisms of laser-tissue interaction: II. Tissue thermal properties. J Lasers Med Sci 4:99-106, 2013

2. Ascher PW, Justich E, Schröttner O: A new surgical but less invasive treatment of central brain tumours. Preliminary report. Acta Neurochir Suppl (Wien) 52:78-80, 1991

3. Attaar SJ, Patel NV, Hargreaves E, Keller IA, Danish SF: Accuracy of laser placement with frameless stereotaxy in magnetic resonance-guided laser-induced thermal therapy. Oper Neurosurg (Hagerstown) 11:554-563, 2015

4. Bettag M, Ulrich F, Schober R, Fürst G, Langen KJ, Sabel M, et al: Stereotactic laser therapy in cerebral gliomas. Acta Neurochir Suppl (Wien) 52:81-83, 1991

5. Carpentier A, Chauvet D, Reina V, Beccaria K, Leclerq D, McNichols RJ, et al: MR-guided laser-induced thermal therapy (LITT) for recurrent glioblastomas. Lasers Surg Med 44:361-368, 2012

6. Carpentier A, McNichols RJ, Stafford RJ, Itzcovitz J, Guichard JP, Reizine D, et al: Real-time magnetic resonance-guided laser thermal therapy for focal metastatic brain tumors. Neurosurgery 63 (1 Suppl 1):ONS21-ONS29, 2008

7. Curry DJ, Gowda A, McNichols RJ, Wilfong AA: MR-guided stereotactic laser ablation of epileptogenic foci in children. Epilepsy Behav 24:408-414, 2012

8. Esquenazi Y, Kalamangalam GP, Slater JD, Knowlton RC, Friedman E, Morris SA, et al: Stereotactic laser ablation of epileptogenic periventricular nodular heterotopia. Epilepsy Res 108:547-554, 2014

9. Goldberg SN, Gazelle GS, Mueller PR: Thermal ablation therapy for focal malignancy: a unified approach to underlying principles, techniques, and diagnostic imaging guidance. AJR Am J Roentgenol 174:323-331, 2000

10. Gonzalez-Martinez J, Vadera S, Mullin J, Enatsu R, Alexopoulos AV, Patwardhan R, et al: Robot-assisted stereotactic laser ablation in medically intractable epilepsy: operative technique. Neurosurgery 10 (Suppl 2):167-173, 2014

11. Jacques SL, Prahl SA: Modeling optical and thermal distributions in tissue during laser irradiation. Lasers Surg Med 6:494-503, 1987

12. Jethwa PR, Barrese JC, Gowda A, Shetty A, Danish SF: Magnetic resonance thermometry-guided laser-induced thermal therapy for intracranial neoplasms: initial experience. Neurosurgery 71 (1 Suppl Operative):133-145, 2012

13. Jethwa PR, Lee JH, Assina R, Keller IA, Danish SF: Treatment of a supratentorial primitive neuroectodermal tumor using magnetic resonance-guided laser-induced thermal therapy. J Neurosurg Pediatr 8:468-475, 2011 
14. Kahn T, Bettag M, Ulrich F, Schwarzmaier HJ, Schober R, Fürst G, et al: MRI-guided laser-induced interstitial thermotherapy of cerebral neoplasms. J Comput Assist Tomogr 18:519-532, 1994

15. Keijzer M, Jacques SL, Prahl SA, Welch AJ: Light distributions in artery tissue: Monte Carlo simulations for finitediameter laser beams. Lasers Surg Med 9:148-154, 1989

16. McGahan JP, Griffey SM, Budenz RW, Brock JM: Percutaneous ultrasound-guided radiofrequency electrocautery ablation of prostate tissue in dogs. Acad Radiol 2:61-65, 1995

17. McNichols RJ, Gowda A, Kangasniemi M, Bankson JA, Price RE, Hazle JD: MR thermometry-based feedback control of laser interstitial thermal therapy at $980 \mathrm{~nm}$. Lasers Surg Med 34:48-55, 2004

18. Medvid R, Ruiz A, Komotar RJ, Jagid JR, Ivan ME, Quencer RM, et al: Current applications of MRI-guided laser interstitial thermal therapy in the treatment of brain neoplasms and epilepsy: a radiologic and neurosurgical overview. AJNR Am J Neuroradiol 36:1998-2006, 2015

19. Mohammadi AM, Hawasli AH, Rodriguez A, Schroeder JL, Laxton AW, Elson P, et al: The role of laser interstitial thermal therapy in enhancing progression-free survival of difficult-to-access high-grade gliomas: a multicenter study. Cancer Med 3:971-979, 2014

20. Motamedi M, Welch AJ, Cheong WF, Ghaffari SA, Tan OT: Thermal lensing in biologic medium. IEEE J Quantum Electron 24:693-696, 1988

21. Munier SM, Hargreaves EL, Patel NV, Danish SF: Effects of variable power on tissue ablation dynamics during magnetic resonance-guided laser-induced thermal therapy with the Visualase system. Int J Hyperthermia 34:764-772, 2018

22. Norred SE, Johnson JA: Magnetic resonance-guided laser induced thermal therapy for glioblastoma multiforme: a review. BioMed Res Int 2014:761312, 2014

23. Patel NV, Frenchu K, Danish SF: Does the thermal damage estimate correlate with the magnetic resonance imaging predicted ablation size after laser interstitial thermal therapy? Oper Neurosurg (Hagerstown) 15:179-183, 2018

24. Pearce JA: Comparative analysis of mathematical models of cell death and thermal damage processes. Int J Hyperthermia 29:262-280, 2013

25. Rao MS, Hargreaves EL, Khan AJ, Haffty BG, Danish SF: Magnetic resonance-guided laser ablation improves local control for postradiosurgery recurrence and/or radiation necrosis. Neurosurgery 74:658-667, 2014

26. Rastegar S, Motamedi M, Welch AJ, Hayes LJ: A theoretical study of the effect of optical properties in laser ablation of tissue. IEEE Trans Biomed Eng 36:1180-1187, 1989

27. Reichert WM (ed): Indwelling Neural Implants: Strategies for Contending with the In Vivo Environment. Boca Raton, FL: CRC Press/Taylor \& Francis, 2008

28. Sinha S, Hargreaves E, Patel NV, Danish SF: Assessment of irrigation dynamics in magnetic-resonance guided laser induced thermal therapy (MRgLITT). Lasers Surg Med 47:273-280, 2015

29. Sugahara T, Korogi Y, Kochi M, Ikushima I, Hirai T, Okuda $\mathrm{T}$, et al: Correlation of MR imaging-determined cerebral blood volume maps with histologic and angiographic determination of vascularity of gliomas. AJR Am J Roentgenol 171:1479-1486, 1998

30. Sun XR, Patel NV, Danish SF: Tissue ablation dynamics during magnetic resonance-guided, laser-induced thermal therapy. Neurosurgery 77:51-58, 2015

31. Thomsen S: Pathologic analysis of photothermal and photomechanical effects of laser-tissue interactions. Photochem Photobiol 53:825-835, 1991

32. Torres-Reveron J, Tomasiewicz HC, Shetty A, Amankulor NM, Chiang VL: Stereotactic laser induced thermotherapy (LITT): a novel treatment for brain lesions regrowing after radiosurgery. J Neurooncol 113:495-503, 2013

33. Vogel A, Venugopalan V: Mechanisms of pulsed laser ablation of biological tissues. Chem Rev 103:577-644, 2003

34. Welch A: The thermal response of laser irradiated tissue. IEEE J Quantum Electron 20:1471-1481, 1984

35. Willie JT, Laxpati NG, Drane DL, Gowda A, Appin C, Hao $\mathrm{C}$, et al: Real-time magnetic resonance-guided stereotactic laser amygdalohippocampotomy for mesial temporal lobe epilepsy. Neurosurgery 74:569-585, 2014

\section{Disclosures}

Dr. Danish is a consultant for Medtronic and has received educational honoraria.

\section{Author Contributions}

Conception and design: Danish, Munier. Acquisition of data: Danish, Munier. Analysis and interpretation of data: Danish, Munier, Patel. Drafting the article: Munier, Hargreaves. Critically revising the article: Danish, Hargreaves, Patel. Reviewed submitted version of manuscript: all authors. Approved the final version of the manuscript on behalf of all authors: Danish. Statistical analysis: Hargreaves. Study supervision: Danish.

\section{Supplemental Information}

\section{Previous Presentations}

Portions of this work were presented at the American Society for Stereotactic and Functional Neurosurgery Biennial Meeting as an oral presentation in Denver, Colorado, on June 2, 2018.

\section{Correspondence}

Shabbar F. Danish: Rutgers University, New Brunswick, NJ. shabbar.danish@rutgers.edu. 